\title{
Réseau de régulation de la transcription des gènes du système protéolytique de Lactococcus lactis
}

\author{
Eric GuÉdon, Cécile Martin, François-Xavier Gobert, \\ S. Dusko Ehrlich, Pierre Renault, Christine Delorme*
}

Laboratoire de Génétique Microbienne, INRA, Domaine de Vilvert, 78352 Jouy-en-Josas Cedex, France

\begin{abstract}
Network of regulation of gene transcription of the proteolytic system of Lactococcus lactis. The proteolytic system of lactococci that allows degradation of caseins and proteins of milk is complex. Milk proteins contain all amino acids necessary for growth of lactic acid bacteria. The proteolytic system consists of an extracellularly located proteinase, transport systems for di-tripeptides and oligopeptides and a multitude of intracellular peptidases. Expression of 13 genes was followed by transcriptional fusions in presence of different peptide sources. Transcription of 6 genes is repressed in media containing peptides and that of 4 genes (pepN, pepC, $p r t P$ and opp-pepOl operon) by dipeptides containing one of the 3 branched amino acids (isoleucine, leucine and valine). Repression of gene transcription required that regulatory peptides are translocated into the cell and degraded in amino acids. Cell factors involved in this regulation were identified in derepressed mutants obtained by random mutagenesis by transposition. DtpT, a di-tripeptides transporter and CodY, homologous of the Bacillus subtilis pleiotropic regulator of transcription were the most frequently inactivated proteins. pepC, pepN and opp-pepOl transcription is not repressed in $\operatorname{cod} Y$ and $d t p T$ mutant. These genes of the proteolytic system belong to a same regulon since their expression is repressed by CodY regulator depending on intracellular concentration of branched amino acids or derivative products of them.
\end{abstract}

Lactococcus lactis / proteolytic system / transcription / regulation

Résumé - Lactococcus lactis possède un système protéolytique complexe pour dégrader les caséines, protéines majoritaires du lait qui contiennent tous les acides aminés nécessaires à sa croissance. Ce système comprend une protéase de paroi extracellulaire, trois systèmes de transport spécifiques des di-tripeptides et des oligopeptides et de nombreuses peptidases localisées à l'intérieur de la cellule. L'influence de la source de peptides sur l'expression de 13 gènes de ce système a été caractérisée grâce à des fusions transcriptionnelles. La transcription de 6 de ces gènes est réprimée en présence d'une source riche en peptides et pour 4 d'entre eux (pepN, pepC, prtP et l'opéron opp-pepOl) par des dipeptides qui contiennent au moins un des trois acides aminés branchés (leucine, isoleucine et valine). Pour permettre la répression de la transcription, les dipeptides doivent être transportés et hydrolysés en acides

* Correspondance et tirés-à-part

Tél. : (33) 134652526 ; fax : (33) 134652521 ; e-mail : delorme @ biotec.jouy.inra.fr 
aminés dans la cellule. Des facteurs cellulaires impliqués dans la régulation de l'expression de ces différents gènes ont été identifiés dans des mutants déréprimés, obtenus par mutagenèse aléatoire par transposition. Il s'agit du transporteur des di- et tripeptides (DtpT) et d'une protéine homologue à la protéine CodY, qui est un régulateur pléiotrope de la transcription de Bacillus subtilis. Nous avons montré que les gènes pepC, pepN et l'opéron opp-pepO1 constituent un régulon dont l'expression est réprimée par CodY en fonction de la concentration intracellulaire en acides aminés branchés ou d'un produit de leur catabolisme.

Lactococcus lactis / système protéolytique / transcription / régulation

\section{INTRODUCTION}

Lactococcus lactis possède un système protéolytique complexe pour dégrader la caséine et les protéines du lait qui contiennent tous les acides aminés nécessaires à sa croissance. La composition et la concentration des acides aminés et des peptides libres du lait ne permettent pas la croissance optimale de L. lactis qui, de plus, est une bactérie auxotrophe pour plusieurs acides aminés $[2,15]$. Le système protéolytique qui contrôle donc la nutrition azotée de L. lactis est également impliqué dans le développement de la saveur et de la flaveur des fromages par la production de peptides et d'acides aminés, précurseurs d'arômes.

Les enzymes de L. lactis intervenant dans la protéolyse ont été largement étudiées sur le plan biochimique (spécificité de substrat, conditions optimales d'activité) permettant ainsi, la caractérisation des principales étapes de la dégradation des caséines. La première étape de ce processus est réalisée par une endoprotéase (PrtP) liée à l'enveloppe bactérienne, qui dégrade les protéines du lait en peptides. Ceux-ci, ainsi que les peptides libres du lait, sont ensuite transportés dans la cellule par 3 systèmes de transport spécifiques (Opp, DtpT et DtpP). Enfin, ces peptides sont hydrolysés en acides aminés par une vingtaine de peptidases localisées dans le cytoplasme (PepA, PepC, PepF1, PepF2, PepN, PepO1, PepO2, PepP, PepQ, PepT, PepV, PepX, Pcp, PepDA1, PepDA2, PepM, PepQ) [1, 3, 5, $7,11]$.
Bien que la plupart des gènes codant pour ces enzymes aient été caractérisés, les études concernant l'expression de ces gènes et leur régulation sont rares chez L. lactis $[1,3,5]$. Les principaux résultats de régulation d'expression de ces gènes concernent un seul des composant du système protéolytique, la protéase de paroi. La transcription du gène codant pour la protéase extracellulaire de L. lactis SK11 est réprimée 10 fois quand la concentration en peptides du milieu augmente [8]. L'addition de dipeptides spécifiques a montré que les dipeptides prolylleucine et leucylproline répriment la transcription du gène $\operatorname{prt} P$ [9]. Le dipeptide prolylleucine pourrait aussi réprimer l'activité de PepN et PepX [10].

Dans ce contexte nous avons caractérisé la régulation de l'expression de la plupart des gènes du système protéolytique de L. lactis. Pour cela, nous avons recherché à identifier à la fois les facteurs environnementaux et les facteurs cellulaires pouvant affecter l'expression des gènes codant pour les composants du système protéolytique de cette bactérie. D'une part, l'influence de la source de peptides a été testée sur l'expression des gènes codant pour des enzymes du système protéolytique. La transcription des gènes codant pour 11 peptidases, pour les protéases de type PI et PIII ainsi que pour un des trois systèmes de transport de peptides de L. lactis a été étudiée grâce à des fusions transcriptionnelles. D'autre part, dans le but d'identifier des facteurs impliqués dans la régulation de l'expression de ces différents gènes, des mutants dont la transcription n'est 
plus soumise à régulation ont été caractérisés par une mutagenèse aléatoire par transposition.

\section{CONSTRUCTION DES FUSIONS TRANSCRIPTIONNELLES}

Quatorze promoteurs ont été fusionnés aux gènes rapporteurs de la luciférase de Vibrio harveyi (luxAB) sur des vecteurs qui permettent l'intégration des fusions sur le chromosome de L. lactis MG1363 [5, 6]. Les 10 promoteurs des gènes chromosomiques рерP, рерA, pерF2, рерDA1, рерQ, рерX, рерM, рерT, рерN, рерC et les deux de l'opéron opp-pepOl [16] (PpepOA et PpepOD) ont été amplifiés par PCR à l'aide d'amorces spécifiques à partir du chromosome de la souche de L. lactis ssp. cremoris MG1363. Les promoteurs des gènes plasmidiques prtPl et prtPIII ont été amplifiés par PCR à l'aide d'amorces spécifiques respectivement à partir des souches de L. lactis ssp. cremoris WG2 et SK11.

Les fragments PCR contenant les différents promoteurs ont été clonés en amont des gènes $\operatorname{lu} x A B$ qui sont dépourvus de leur propre promoteur, soit dans des plasmides intégratifs (pJIM2374, pVar-1) pour ceux contenant les promoteurs de gènes chromosomiques, soit dans un plasmide réplicatif (pJIM2366) pour ceux contenant les promoteurs de gènes plasmidiques [12]. Les deux vecteurs intégratifs, à réplication conditionnelle, ont été utilisés pour intégrer les fusions transcriptionnelles sur le chromosome. Le pJIM2374 permet d'intégrer chaque fusion par recombinaison homologue à son locus promoteur dans la souche MG1363 [5]. Le pVar-1 qui contient un fragment du facteur sexuel de la MG1363 permet de diriger l'intégration dans cet élément chromosomique [6]. Le facteur sexuel est transféré par conjugaison à des taux de $10^{-3}-10^{-6}$ entre souches de lactocoques [4]. Ainsi, les fusions intégrées dans le facteur sexuel à l'aide du pVar-1, ont été transférées par conjugaison aux souches mutantes
MG1363 dtpT, pep $Q$ et $\operatorname{cod} Y$. La caractérisation de l'intégration au locus voulu se fait grâce à des amorces appropriées par amplification PCR et par hybridation d'ADN de type Southern.

Les mesures d'activité luciférase sont effectuées sur le luminomètre Bertold Lumat LB9501. Un millilitre de culture de L. lactis est mélangé avec $5 \mu \mathrm{L}$ de nonaldehyde et l'émission de lumière est directement mesurée. La valeur du pic est ramenée à la $\mathrm{DO}_{600 \mathrm{~nm}}$ de la culture. L'activité luciférase est mesurée tout au long de la croissance. L'activité luciférase reportée dans les différentes figures est mesurée à $\mathrm{DO}_{600 \mathrm{~nm}} 0,4$ et exprimée en lux/DO $\left[10^{3}\right]$.

\section{EFFET DE LA SOURCE DE PEPTIDES SUR L'EXPRESSION DES GÈNES DE LA PROTÉOLYSE}

\subsection{Effet de sources riches en peptides}

La transcription des gènes pepA, рерC, рерDA1, рерF2, рерM, рерN, рерP, рерQ, pepT, pepX, prtPl, prtP3 et de l'opéron opppepOl a été analysée à l'aide de 14 fusions lux. Cette analyse a été menée en milieu chimiquement défini (MCD) lors de la croissance des souches contenant les fusions en présence de différentes sources de peptides. Le MCD [14] contient pour source d'azote un mélange de 18 acides aminés suffisant pour la croissance des lactocoques et il a été supplémenté soit par des solutions de casitones $(1,5 \%)$, de casa-aminoacides (CAA à $1,5 \%)$ ou des dipeptides synthétiques (leucylproline [LP] et prolylleucine [PL] à $\left.1 \mathrm{mmol} \cdot \mathrm{L}^{-1}\right)$. Les casitones ( $80 \%$ peptides, $20 \%$ d'acides aminés) et les CAA (20\% peptides, $80 \%$ d'acides aminés) sont respectivement des hydrolysats enzymatique et acide des caséines qui sont les protéines majoritaires du lait.

Les mesures luciferases de la fusion Ppe$p O D$-lux obtenues après croissance bactérienne avec ces différentes sources de 
peptides sont représentées dans la figure 1 . L'expression de cette fusion qui correspond à l'expression du premier promoteur de l'opéron opp-pepO1 est maximale en MCD. Cette expression est réprimée $45 ; 6,5 ; 4$ et 4,5 fois respectivement par la source de casitones, la source de CAA, les dipeptides LP et PL (Fig. 1). La source de casitones qui contient 4 fois plus de peptides que les CAA, réprime 7 fois plus l'expression de l'opéron opp-pepOl que la source de CAA. La répression de la transcription de l'opéron opp-pepOl est dépendante de la source de peptides.

Les fusions avec les 14 autres promoteurs ont également une activité plus importante en MCD qu'en présence d'une source de peptides. Les 14 promoteurs ont ainsi été classés d'après le niveau d'expression obtenue en MCD sur la figure 2. Les expressions les plus fortes en MCD ( 1 à $5 \times 10^{3} \mathrm{lux} /$ $\left.\mathrm{DO}\left[10^{3}\right]\right)$ correspondent à celles des gènes prtPI, prtPIII pepC, pepN et l'opéron opp-pepO1. La transcription de ces gènes codant pour des protéines ayant un rôle important dans l'utilisation de la caséine est réprimé de 7 à 150 fois en présence de casitones (Fig. 2). La transcription des gènes рерX, рерM, рерT, рер $Q$, pерDA1, рерF2, рерA et pepP en MCD est beaucoup plus faible (200 à 20 lux/DO [10 $\left.\left.30^{3}\right]\right)$. En présence de casitones, seule l'expression de pepX est réprimée 5 fois. La faible diminution de l'expression des autres promoteurs en présence de casitones (< à 3) ne sera pas considérée comme significative dans cette étude [5].

\subsection{Effet de dipeptides défini}

Pour caractériser les signaux présents dans la source de peptides, qui conduisent à la répression de la transcription de ces gènes, nous avons caractérisé l'effet de 65 dipeptides sur l'expression de l'opéron opppepO1. Deux classes de dipeptides ont été définies d'après leur effet sur l'expression de la fusion contenant le promoteur PpepOD. Les dipeptides de la classe 1 n'ont pas d'effet sur l'expression de la fusion PpepOD-lux alors que ceux de la classe 2

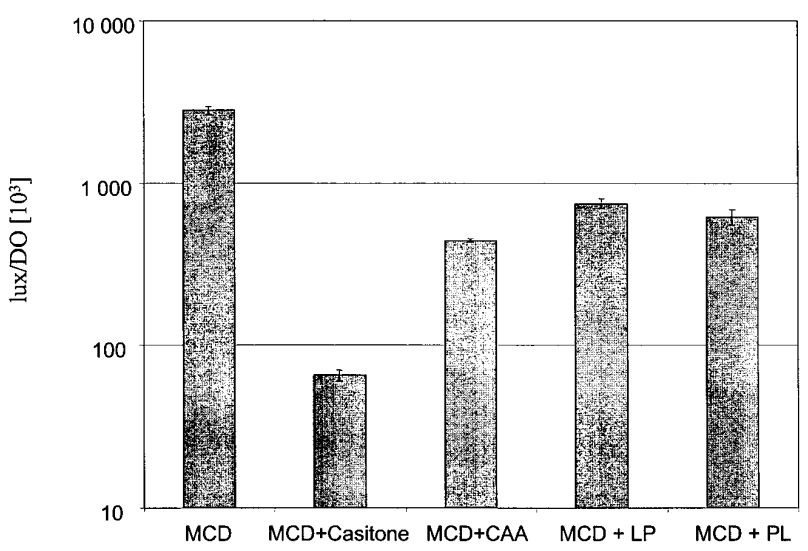

Figure 1. Activités luciférases de L. lactis MG1363 contenant la fusion PpepOD-lux et cultivé en MCD seul ou supplémenté avec CAA, casitone ou les dipeptides LP ou PL. Les barres verticales représentent l'écart type des mesures. L'activité luciférase est mesurée à $\mathrm{DO}_{600 \mathrm{~nm}}=0,4$ et exprimée en lux/DO $\left[10^{3}\right]$.

Figure 1. Luciferase activities of L. lactis MG1363 harboring the PpepOD-lux fusion and cultivated on CDM and on CDM supplemented with CAA, casitone or LP or PL dipeptides. Vertical bars represent the standard deviation of the measurements. Luciferase activity was measured at $\mathrm{DO}_{600 \mathrm{~nm}}=$ 0.4 and is expressed in lux/OD [10 $\left.{ }^{3}\right]$. 


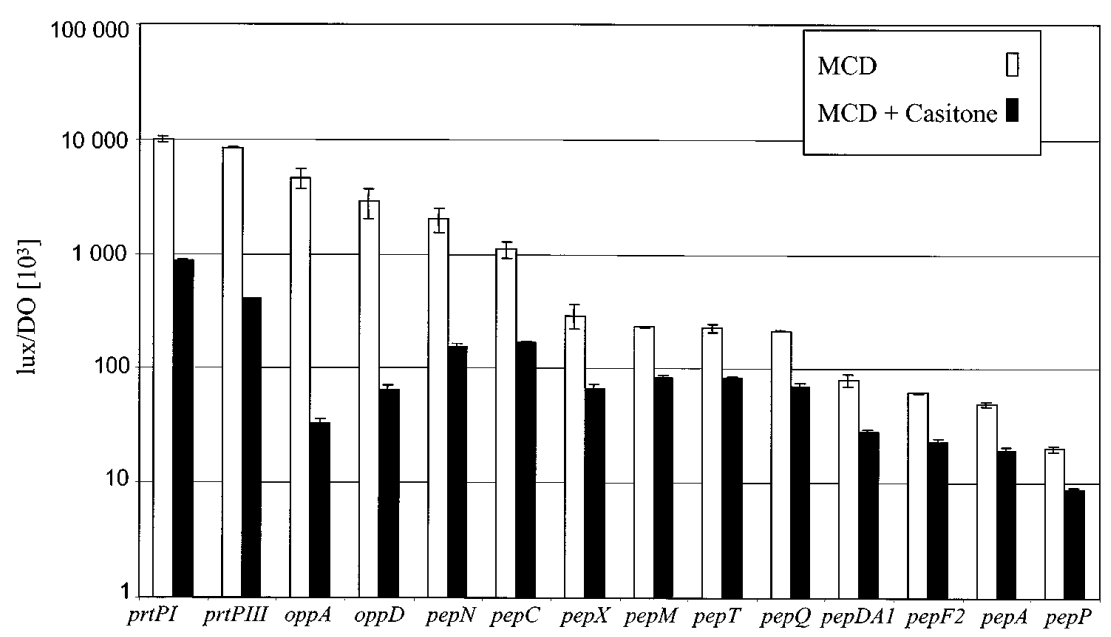

Figure 2. Activités luciférases de L. lactis MG1363 contenant les 14 fusions lux avec les promoteurs de gènes codant pour des enzymes de système protéolytique. Les cellules sont cultivées en MCD (barres blanches) et MCD avec casitone (barres noires). Les barres verticales représentent l'écart type des mesures. L'activité luciférase est mesurée à $\mathrm{DO}_{600 \mathrm{~nm}}=0,4$ et exprimée en lux/DO $\left[10^{3}\right]$.

Figure 2. Luciferase activities of L. lactis MG1363 harboring the 14 lux fusions with promoters of genes coding for enzymes of the proteolytic system. Cells were grown in CDM (white bars) and CDM supplemented with casitone (black bars). Vertical bars represent the standard deviation of the measurements. Luciferase activity was measured at $\mathrm{DO}_{600 \mathrm{~nm}}=0.4$ and is expressed in lux/OD $\left[10^{3}\right]$.

répriment 4 à 7 fois l'expression de cette fusion (Fig. 3) [5]. Parmi les 65 dipeptides testés, les 33 dipeptides régulateurs qui répriment la transcription de l'opéron opp-pepOI contiennent un des 3 acides aminés branchés (leucine, isoleucine et valine). Les dipeptides non régulateurs ne contiennent pas d'acides aminés branchés. Les dipeptides régulateurs répriment également l'expression des gènes pepN, pepC et $p r t P$ alors qu'ils n'ont aucun effet régulateur sur la transcription du gène $p e p X$ [5]. Il est ainsi possible de définir un régulon contenant les gènes pepN, pep $C, p r t P$ et opp-pepOI dont la transcription est réprimée par les dipeptides contenant des ILV.

\subsection{Le signal régulateur est intracellulaire}

Pour déterminer si les dipeptides régulateurs ont une action extra- ou intracellulaire, l'expression de l'opéron opp-pepOl a été caractérisée dans un mutant de transport des dipeptides. L. lactis possède 2 systèmes de transport des di-tripeptides, DtpP et DtpT. Il a été vérifié que les dipeptides régulateurs KL et TL n'étaient plus transportés dans un mutant $d t p T$ à l'inverse du dipeptide $\mathrm{LV}$. La fusion PpepOD-lux a été transférée dans un mutant $d t p T$ et son expression a été analysée après croissance bactérienne en MCD supplémenté avec un des 3 dipeptides suivants, TL, KL et LV (Fig. 4A). Ces 3 dipeptides répriment 4,5 à 8 fois l'expression de l'opéron opp-pepO1 dans la souche sauvage MG1363. Dans le mutant $d t p T$, l'expression de l'opéron opp-pepO1 est toujours réprimée par le dipeptide LV alors qu'elle ne l'est plus par les dipeptides non transportés KL et TL. Les dipeptides régulateurs exercent un rôle répresseur seulement après leur entrée dans la cellule [5]. 


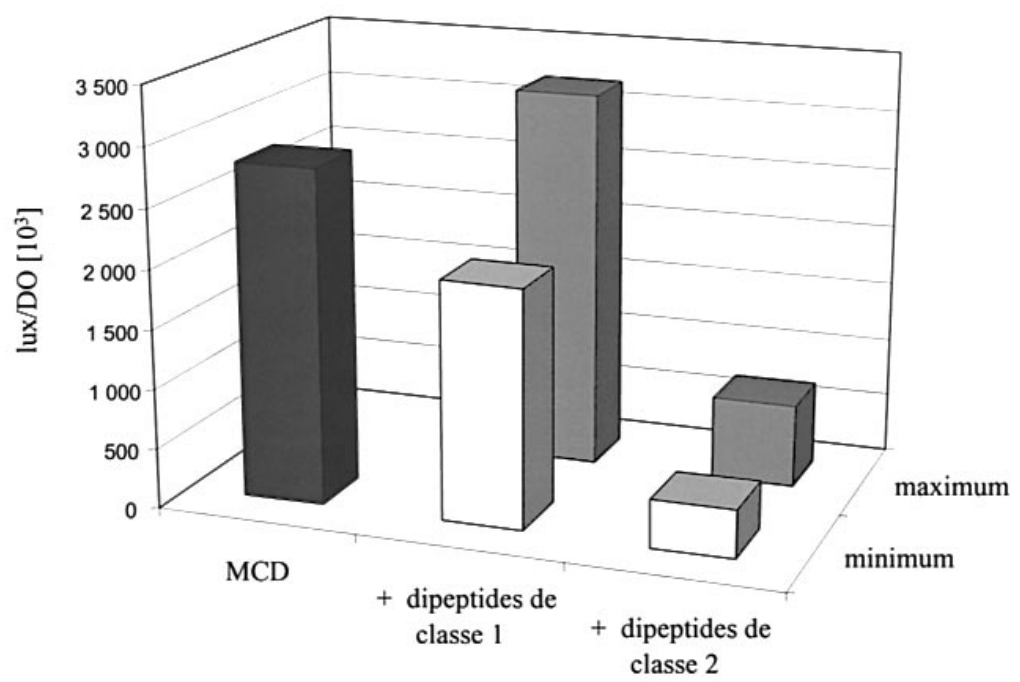

Figure 3. Activités luciférases de L. lactis MG1363 contenant la fusion PpepOD-lux et cultivé en MCD et en MCD supplémenté avec différents dipeptides. L'activité luciférase en MCD est représentée par une barre noire. En MCD supplémenté avec différents dipeptides, l'activité luciférase minimum est représentée par les barres blanches et l'activité luciférase maximale est représentée par les barres grises. La classe 1 est composée des dipeptides suivants : FA, FM, FP, PP, PA, PY, ES, FG, DQ, CG, WE, HK, RK, TR, YA, MA, HP, WA, DA, DK, PF, MP, SY, SE, AF, FF, PM, AP, AA, TQ, AF, GN. La classe 2 est composée des dipeptides suivants : AL, LA, LL, LM, LV, FV, PL, VA, VM, VF, VV, LF, VP, LP, GI, RI, HL, LD, LG, LN, VQ, AI, VL, AV, KL, WL, GV, IN, TL, VS, EV, YL, FL. L'activité luciférase est mesurée à $\mathrm{DO}_{600 \mathrm{~nm}}=0,4$ et exprimée en lux/DO $\left[10^{3}\right]$.

Figure 3. Luciferase activities of L. lactis MG1363 harboring PpepOD-lux fusion and cultivated on $\mathrm{CDM}$ and on CDM supplemented with several dipeptides. Black bar represents luciferase activity in CDM. In CDM supplemented with several dipeptides, white bars represent the minimum of luciferase activity and grey bars represent the maximum of luciferase activity. Vertical bars represent the standard deviation of the measurements. Dipeptides of class 1: FA, FM, FP, PP, PA, PY, ES, FG, DQ, CG, WE, HK, RK, TR, YA, MA, HP, WA, DA, DK, PF, MP, SY, SE, AF, FF, PM, AP, AA, TQ, AF, GN. Dipeptides of class 2 : AL, LA, LL, LM, LV, FV, PL, VA, VM, VF, VV, LF, VP, LP, GI, RI, HL, LD, LG, LN, VQ, AI, VL, AV, KL, WL, GV, IN, TL, VS, EV, YL, FL. Luciferase activity was measured at $\mathrm{DO}_{600 \mathrm{~nm}}=0.4$ and is expressed in lux/OD $\left[10^{3}\right]$.

\subsection{Le signal régulateur est un produit de dégradation des dipeptides}

Pour déterminer si le signal intracellulaire de répression de la transcription est les dipeptides ou un des produits de leur catabolisme, l'expression de l'opéron opppepO1 a été caractérisée dans un mutant pep $Q$ incapable de dégrader certains dipeptides. Chez L. lactis, la dipeptidase PepQ dégrade spécifiquement les dipeptides de type X-Pro. Il a été vérifié que les dipeptides régulateurs LP et VP ne sont plus hydrolysés dans le mutant pep $Q$ à l'inverse du dipeptide PL. L'expression de la fusion PpepOD-lux transférée dans le mutant pep $Q$ a été analysée après croissance bactérienne en MCD supplémenté avec un des trois dipeptides LP, VP ou PL (Fig. 4B). Ces dipeptides répriment 8 à 8,5 fois l'expression de l'opéron opp-pepOl dans la souche sauvage MG1363. Chez un mutant pepQ, l'expression de l'opéron opp-pepOl est 


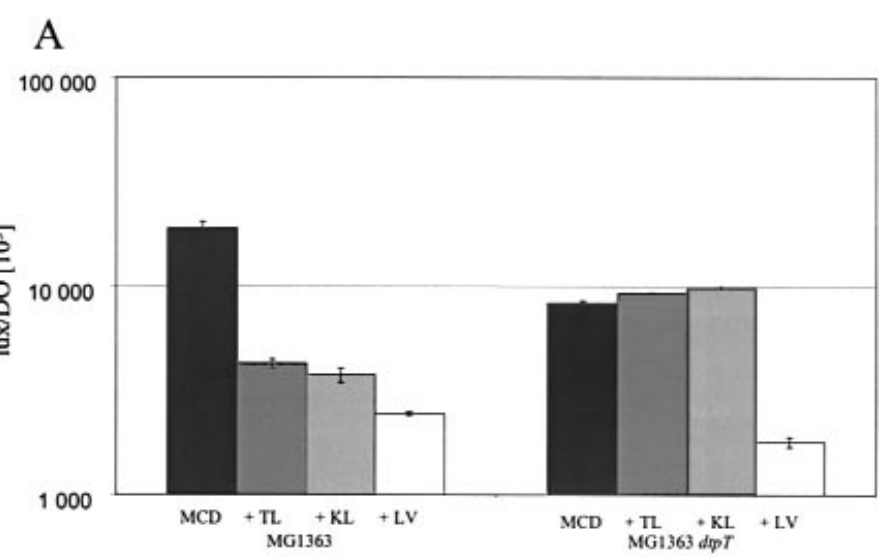

B

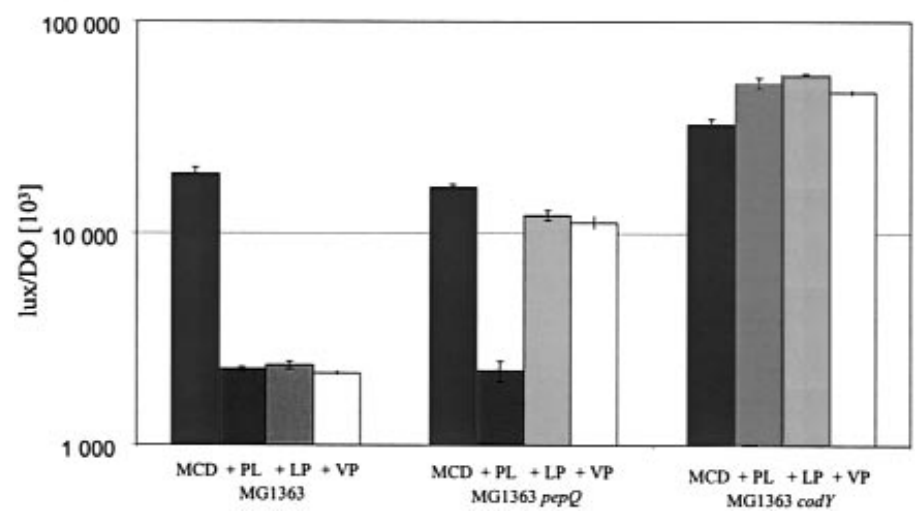

Figure 4. Activités luciférases de L. lactis MG1363 et des souches mutantes $d t p T$ (A), pepQ et $\operatorname{codY}(\mathbf{B})$ contenant la fusion PpepOD-lux. Les cellules ont été cultivées en MCD et MCD supplémenté avec différents dipeptides. Les barres verticales représentent l'écart type des mesures. L'activité luciférase est mesurée à $\mathrm{DO}_{600 \mathrm{~nm}}=0,4$ et exprimée en lux/DO $\left[10^{3}\right]$.

Figure 4. Luciferase activities of L. lactis MG1363 and mutant strains $\operatorname{dtpT}(\mathbf{A})$, pep $Q$ and $\operatorname{codY}(\mathbf{B})$ harboring the PpepOD-lux fusion. Cells were grown on CDM and CDM supplemented with different dipeptides. Vertical bars represent the standard deviation of the measurements. Luciferase activity was measured at $\mathrm{DO}_{600 \mathrm{~nm}}=0.4$ and is expressed in lux/OD $\left[10^{3}\right]$.

toujours réprimée par le dipeptide PL alors qu'elle ne l'est plus par les dipeptides LP et VP qui ne sont pas hydrolysés. Les dipeptides non hydrolysés dans le mutant pep $Q$ n'ont plus d'effet répresseur ce qui indique que les dipeptides n'ont pas un rôle répresseur par eux-mêmes. Un produit du catabolisme des peptides régulateurs réprime donc la transcription de l'opéron opp-pepOl [5].

\section{RECHERCHE DE COMPOSANTS CELLULAIRES IMPLIQUÉS DANS LA RÉGULATION PAR LES PEPTIDES DU MILIEU}

Dans le but d'identifier des protéines impliquées dans la régulation de l'expression de ces différents gènes, une mutagenèse aléatoire par transposition a été effectuée. 
Elle a permis d'identifier des mutants dont l'expression de l'opéron opp-pepOl est fortement déréprimée lors de la croissance en présence d'une source de peptides. Ces mutants présentent des insertions dans 8 loci chromosomiques différents ; $20 \%$ d'entre eux présentent une insertion du transposon dans le gène $d t p T$ confirmant ainsi l'importance du transport des dipeptides dans cette régulation ; $36 \%$ des mutants présentent une insertion du transposon dans un gène dont le produit présente $48 \%$ d'identité avec la protéine $\operatorname{CodY}$ de $B$. subtilis, un régulateur pléiotrope de la transcription. Chez B. subtilis, la protéine CodY est impliquée dans la répression de la transcription d'une dizaine de gènes dont l'opéron $d p p$ codant pour le système de transport des dipeptides [13].

La fusion PpepOD-lux a été transférée dans le mutant $\operatorname{cod} Y$ et son expression a été analysée après croissance bactérienne en MCD supplémenté avec un dipeptide PL, LP ou VP (Fig. 4B). Ces dipeptides répriment de 8 à 8,5 fois l'expression de l'opéron opp-pepO1 dans la souche sauvage MG1363. Dans un mutant $\operatorname{cod} Y$, l'expression de l'opéron opp-pepO1 n'est plus réprimée par ces 3 dipeptides. Ces résultats montrent d'une part que la protéine CodY est bien impliquée dans la répression de la transcription de l'opéron opp-pepOl et d'autre part, que le pool intracellulaire d'acides aminés branchés ou un produit de leur catabolisme pourrait moduler l'activité de cette protéine. L'expression des gènes pepC et pepN est également contrôlée par la protéine CodY.

\section{CONCLUSION}

Les résultats de cette étude nous ont permis de mettre en évidence que l'expression des gènes prtPI, prtPIII, pepC, pepN, pepX et opp-pepOl est diminuée d'un facteur 5 à 150 par la présence de peptides dans le milieu alors que celle des 7 autres gènes testés n'est pas ou peu affectée. Les gènes dont l'expression est soumise à régulation, codent pour des enzymes clés de la nutrition azotée en lait telles que l'endoprotéase PrtP, les 2 aminopeptidases générales PepN et PepC, l'endopeptidase PepO1 et le système de transport des oligopeptides Opp. L'étude systématique de peptides régulateurs a révélé que des dipeptides contenant un des 3 acides aminés branchés, l'isoleucine, la leucine et la valine (ILV), réprimaient la transcription des gènes prtP, pepC, pepN et opp-pepO1. De plus, l'entrée des dipeptides dans la cellule puis leur dégradation est nécessaire pour qu'ils exercent leur effet répresseur sur la transcription de ces gènes. Ce travail permet de définir un nouveau régulon chez L. lactis, comprenant au moins 4 gènes du système protéolytique dont l'expression est réprimée en présence des produits de dégradation des peptides contenant des ILV. L'expression des gènes de ce régulon serait réprimée par la protéine CodY.

L'ensemble de ces résultats nous permet de proposer un modèle de régulation des gènes du régulon de L. lactis codant pour des enzymes du système protéolytique (Fig. 5). Dans ce modèle, DtpT, en transportant les peptides contenant des ILV, interviendrait donc indirectement en modulant la concentration cytoplasmique en ILV. La protéine CodY aurait un rôle régulateur direct par analogie à son homologue présent chez B. subtilis. Son activité de répresseur de la transcription serait modulée en fonction de la présence d'acides aminés branchés intracellulaires ou de produits de leur dégradation. Cette régulation pourrait permettre d'adapter de manière coordonnée l'expression des gènes impliqués dans l'assimilation des protéines du lait.

De plus, des études préliminaires menées sur l'expression des gènes du régulon lors de croissances bactériennes en lait sont en accord avec le modèle présenté ci-dessus. En effet, durant la phase exponentielle de croissance en lait, les activités luciferases mesurées sont identiques à celles obtenues en MCD alors que durant la phase stationnaire, les activités luciferases mesurées sont 


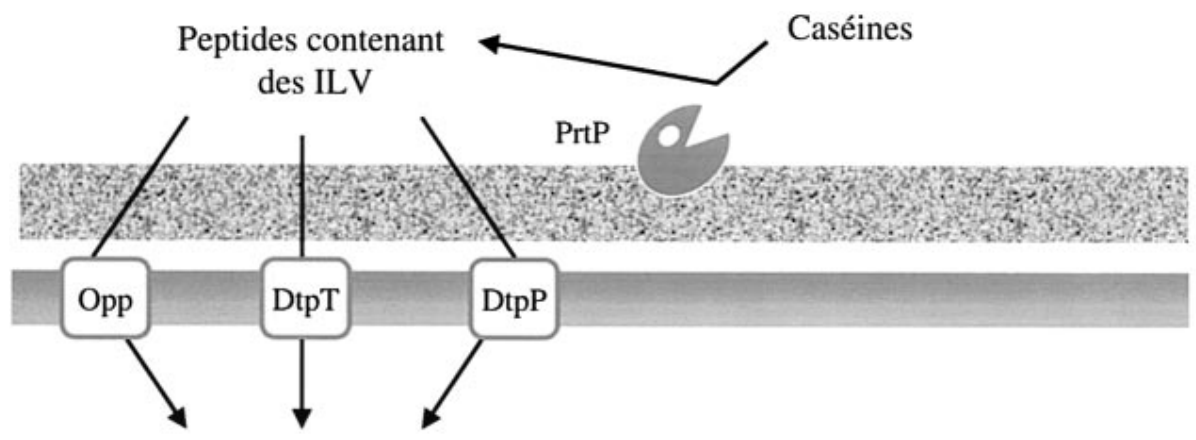

Peptides contenant des ILV

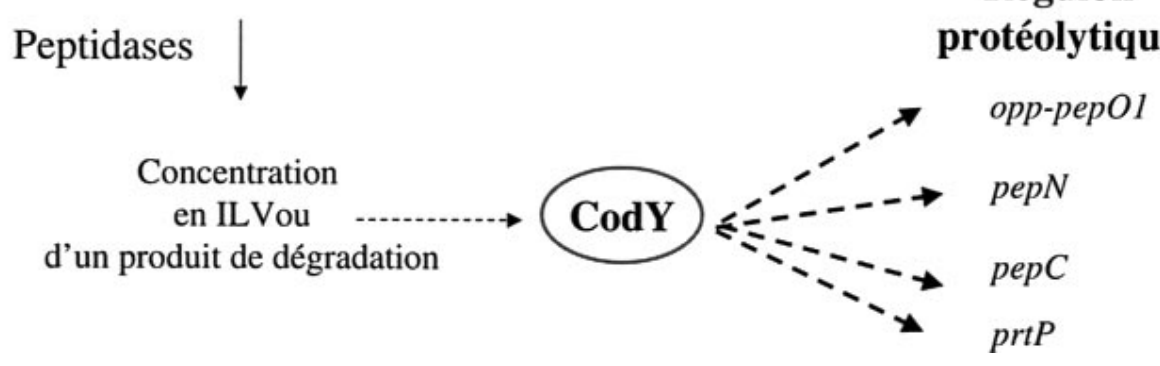

Figure 5. Modèle présentant la régulation de l'expression des gènes du régulon protéolytique de L. lactis.

Figure 5. Model of regulation of gene expression belonging to the proteolytic regulon of L. lactis.

diminuées d'un facteur 2 à 3 . Il peut être envisagé qu'en début de croissance bactérienne, le milieu lait est pauvre en peptides et l'expression des gènes du régulon est maximale. Lors de la dégradation des caséines, le milieu s'enrichit en peptides et ceux-ci alimentent le pool intracellulaire des acides aminés branchés ou d'un produit de leur dégradation, qui serait le signal intracellulaire d'une abondance en peptides du milieu. Dans ces conditions, l'expression des gènes du régulon serait réprimée par CodY et cette régulation coordonnée permettrait donc d'adapter l'expression du régulon en fonction des besoins cellulaires.

En principe, chez un mutant $\operatorname{cod} Y$, les activités peptidolytique et protéolytique ne sont plus réprimées par les peptides issus des caséines. De telles souches pourraient donc être utilisées avantageusement dans certaines fabrications fromagères. L'augmentation des activités peptidolytique et protéolytique d'une souche pourrait permettre d'améliorer l'affinage de certains fromages et ainsi de modifier leur saveur et leur flaveur.

\section{RÉFÉRENCES}

[1] Bolotin A., Mauger S., Malarme K., Ehrlich S.D., Sorokin A., Low-redundancy sequencing of the entire Lactococcus lactis IL1403 genome, Antonie van Leeuwenhoek 76 (1999) 27-76.

[2] Chopin A., Organization and regulation of genes for amino acid biosynthesis in lactic acid bacteria, FEMS Microbiol. Rev. 12 (1993) 21-37.

[3] Christensen J.E., Dudley E.G., Pederson J.A., Steele J.L., Peptidases and amino acid catabolism in lactic acid bacteria, Antonie van Leeuwenhoek 76 (1999) 217-246. 
[4] Godon J.J., Pillidge C.J., Jury K., Gasson M.J., Caractérisation d'un élément conjugatif original : le facteur sexuel de Lactococcus lactis 712 . Lait 76 (1996) 41-49.

[5] Guédon E., Régulation de l'expression des gènes codant pour les composants du système protéolytique chez Lactococus lactis, Thèse de Doctorat de l'université d'Orsay, 2000.

[6] Guédon E., Renault P., Ehrlich S.D., Delorme C., Evaluation de la diversité de l'expression génétique chez les lactocoques : développement d'un outil et son application aux peptidases, Sci. Aliment 20 (2000) 55-62

[7] Kunji E.R., Mierau I., Hagting A., Poolman B., Konings W.N., The proteolytic systems of lac tic acid bacteria, Antonie van Leeuwenhoek 70 (1996) 187-221.

[8] Marugg J.D., Meijer W., van Kranenburg R., Laverman P., Bruinenberg P.G., de Vos W.M., Medium-dependent regulation of proteinase gene expression in Lactococcus lactis: control of transcription initiation by specific dipeptides, J. Bacteriol. 177 (1995) 2982-2989.

[9] Marugg J.D., van Kranenburg R., Laverman P., Rutten G.A., de Vos W.M., Identical transcriptional control of the divergently transcribed prtP and $p r t M$ genes that are required for proteinase production in Lactococcus lactis SK11, J. Bacteriol. 178 (1996) 1525-1531.
[10] Meijer W., Marugg J.D., Hugenholtz J., Regulation of proteolytic enzyme activity in Lactococcus lactis, Appl. Environ. Microbiol. 62 (1996) 156-161.

[11] Pritchard G.G., Coolbear T., The physiology and biochemistry of the proteolytic system in lactic acid bacteria, FEMS Microbiol. Rev. 12 (1993) 179-206.

[12] Renault P., Corthier G., Goupil N., Delorme C. Ehrlich S.D., Plasmid vectors for Gram-positive bacteria switching from high to low copy number, Gene 183 (1996) 175-182.

[13] Serror P., Sonenshein A.L., Interaction of CodY a novel Bacillus subtilis DNA-binding protein with the $d p p$ promoter region, Mol. Microbiol. 20 (1996) 843-852.

[14] Sissler M., Delorme C., Bond J., Ehrlich S.D., Renault P., Francklyn C., An aminoacyl-tRNA synthetase paralog with a catalytic role in histidine biosynthesis, Proc. Natl. Acad. Sci. USA 96 (1999) 8985-8990.

[15] Thomas T.D., Mills O.E., Proteolytic enzymes of starter bacteria, Neth. Milk Dairy J. 35 (1981) 255-273.

[16] Tynkkynen S., Buist G., Kunji E., Kok J. Poolman B., Venema G., Haandrikman A., Genetic and biochemical characterization of the oligopeptide transport system of Lactococcus lactis, J. Bacteriol. 175 (1993) 7523-7532. 